\title{
On $p$-minimal homological models of twisted tensor products of elementary complexes localised over a prime
}

\author{
J. Andrés Armario, Pedro Real \\ and Beatriz Silva \\ Dpto. de Matemática Aplicada I \\ Univ. de Sevilla, 41012 (Spain) \\ e-mail: armario@cica.es, real@cica.es \\ and silva@cica.es
}

\begin{abstract}
In this paper, working over $\mathbb{Z}_{(p)}$ and using algebra perturbation results from [18], p-minimal homological models of twisted tensor products (TTPs) of Cartan's elementary complexes are obtained. Moreover, making use of the notion of indecomposability of a TTP, we deduce that a homological model of a indecomposable $p$-minimal TTP of length $\ell(\ell \geq 2)$ of exterior and divided power algebras is a tensor product of $k$-indecomposable $(k \leq \ell) p$-minimal TTPs of exterior and divided power algebras.
\end{abstract}

\section{Introduction}

Henri Cartan described in his study [3] of the homology of Eilenberg-Mac Lane spaces four types of commutative differential graded augmented algebras, called elementary complexes, which can be made by using exterior and divided power algebras. The first is the exterior algebra $E(u, 2 n-1)$ with one generator $u$ of degree $2 n-1$; the second is the divided power algebra $\Gamma(v, 2 n)$ with one "generator" $v$ of degree $2 n$; the third is the twisted tensor product $\Gamma(u, 2 n) \tilde{\otimes}^{\rho_{ \pm p^{r}}} E(v, 2 n+1)$ with differential operator defined by $\rho_{ \pm p^{r}}(v)= \pm p^{r} u$; and the fourth is the twisted tensor product $E(u, 2 n-1) \tilde{\otimes}^{\rho_{ \pm p^{r}}} \Gamma(v, 2 n)$ with differential operator defined by $\rho_{ \pm p^{r}}(v)= \pm p^{r} u$. Using Cartan's notion of construction, John C. Moore established in [14] and [15] that the $p$-local homology of a DGA-algebra $A$ of this kind is determined in its turn by a tensor product of Cartan's elementary complexes; more precisely, he described the corresponding acyclic Cartan's construction $A \otimes \bar{X}$. A short account of Moore's work on this subject is given by Stasheff in [19]. 
The relation of the methods of the Séminaire Cartan to Homological Perturbation Theory is studied in [10], [11] and, particularly in [12]. In the light of the work of Prouté [16], in which every small Cartan's construction $X=A \otimes \bar{X}$ is described in a concise way (exploiting systematically the notion of Brown's cochain), the fact that this small resolution splits off of the bar resolution $B(A)=A \otimes \bar{B}(A)$ (see [11]) appears as a natural result. The main technique used in [12] is the determination of general recursive formulas (a constructive version of the comparison theorem for resolutions) for a splitting of the bar construction for this class of resolutions.

Another way to obtain the previous splitting from $B(A)$ to $X$ is working $\grave{a}$ la Eilenberg-Mac Lane, that is, handling the reduced bar construction $\bar{B}(A)$ instead of the bar resolution $B(A)$ ). Taking into account Theorem 4.11 of the present paper (a graded-commutative version of Theorem 8.1.3 of [11]), this method can be directly translated to resolutions without any problem. This will be our modus operandi of this paper. We work over $\mathbb{Z}$ localised over a prime $p$ and consider algebra structures and transference problems. The explicit homotopy equivalences from reduced bar constructions of Cartan's elementary complexes to tensor products of DGA-algebras of the same type given in [17] and [18] preserve algebra structures and characterize the particular homological process detailed by Moore in [15]. These results are our point of departure.

We introduce here the algebraic notion of indecomposable twisted tensor product (TTP) of length $\ell$ of commutative DGA-algebras. The elementary complexes can be seen, in particular, as indecomposable TTPs of length 1 and 2 of exterior and divided power algebras. In this way, Moore's method can be considered as a $p$-local homological preservation result of 2-indecomposability. On the other hand, since we work over $\mathbb{Z}_{(p)}$, $\mathbb{Z}$ localised over a prime $p$, we simplify our study using the notion of $p$-minimal DGAalgebra.

Finally, always taking $\mathbb{Z}_{(p)}$ as the ground ring and applying some algebra perturbation results ([18]) and basis change arguments, we prove here a result guaranteeing that a $p$-minimal homological model of a $\ell$-indecomposable (with $\ell \geq 2$ ) $p$-minimal TTP of exterior and divided power algebras is given by a tensor product of $k$-indecomposable (with $k \leq \ell$ ) $p$-minimal TTP of exterior and divided power algebras. In other words, this conclusion, via Theorem 4.11, can be thought as a generalization of the Moore's work in [15].

\section{Preliminaries}

Let $\Lambda$ be a commutative ring with $1 \neq 0$. In this paper, our main objects are commutative connected DGA-algebras.

The differential, product, augmentation and coaugmentation of a DGA-algebra $A$ will be denoted by $d_{A}, \mu_{A}, \epsilon_{A}$ and $\eta_{A}$, respectively. In what follows, the Koszul sign conventions will be used. A morphism $\rho: A_{*} \rightarrow A_{*-1}$ is called derivation if it is 
compatible with the algebra structures on $A$. The degree of an element $a \in A$ is denoted by $|a|$.

We need here the reduced bar construction $\bar{B}(A)$ of a commutative DGA-algebra $A$ (see [13]). Recall that, as a coalgebra, $\bar{B}(A)=T^{c}\left(\Sigma\left(\operatorname{Ker} \epsilon_{A}\right)\right)$ ), where $T^{c}()$ is the tensor coalgebra and $\Sigma()$ is the suspension functor. The element of $\bar{B}_{0}(A)$ corresponding to the identity element of $\Lambda$ is denoted by [ ] and the element $a_{1} \otimes \cdots \otimes a_{n}$ of $\bar{B}(A)$ is denoted by $\left[a_{1}|\cdots| a_{n}\right]$. The tensor and simplicial degrees of the element $\left[a_{1}|\cdots| a_{n}\right]$ are $\left|\left[a_{1}|\cdots| a_{n}\right]\right|_{t}=\sum\left|a_{i}\right|$ and $\left|\left[a_{1}|\cdots| a_{n}\right]\right|_{s}=n$, respectively; its total degree is the sum of its tensor and simplicial degree. A product $*$ (called shuffle product) can be defined on $\bar{B}(A)$, such that the reduced bar construction has a Hopf algebra structure. Recall that the homology of a commutative DGA-algebra $A$ is defined as the homology of the DGA-module $\bar{B}(A)$.

A resolution of $\Lambda$ over the DGA-algebra $A$ is a differential $A$-module $X$ which is projective as an $A$-module and such that the homology of $X$ is zero except in degree 0 where it is $\Lambda$. If $X$ is actually a free $A$-module, then $X$ is called a free resolution.

An example of a free resolution of $\Lambda$ over a DGA-algebra $A$ is the bar resolution (or one-sided bar construction) $B(A)$ ([13], [11]). More concretely, $B(A)$ is the twisted tensor product ([2]) $A \otimes_{\theta} \bar{B}(A)$, where the twisting cochain $\theta$ is given by

$$
\theta\left(\left[a_{1}|\cdots| a_{n}\right]\right)= \begin{cases}a_{1} & n=1 \\ 0 & \text { otherwise }\end{cases}
$$

We deal with a special type of homotopy equivalence. A contraction (see [5], [9]) is a data set $r:\{N, M, f, g, \phi\}$ where $f: N \rightarrow M, g: M \rightarrow N$ are morphisms of DGAmodules (called projection and inclusion, respectively) and $\phi: N \rightarrow N$ is a morphism of graded modules of degree +1 (called the homotopy operator), and these data are required to satisfy the rules: $(\mathbf{r} 1) f g=1_{M},(\mathbf{r} 2) f \phi=0 ;(\mathbf{r} 3) \phi g=0,(\mathbf{r} 4) \phi d_{N}+$ $d_{N} \phi+g f=1_{N}$ and (r5) $\phi \phi=0$.

For instance, the bar resolution $B(A)$ of a DGA-algebra $A$ supports the following contraction:

$$
R_{B(A)}:\left\{B(A), \Lambda, \epsilon_{B(A)}, \eta_{B(A)}, s\right\}
$$

where the homotopy operator $s$ is given by

$$
\begin{gathered}
s: B(A) \rightarrow B(A) \\
s\left(a \otimes\left[a_{1}|\cdots| a_{n}\right]\right)=1 \otimes\left[a\left|a_{1}\right| \cdots \mid a_{n}\right] .
\end{gathered}
$$

A free resolution $K$ of $\Lambda$ over the DGA-algebra $A$ splits off of the bar construction (see [12]) if there is a contraction (called the splitting) from $B(A)$ to $K$. 
We recall the concept of a perturbation datum. Let $N$ be a graded module and let $f: N \rightarrow N$ be a morphism of graded modules. The morphism $f$ is pointwise nilpotent if, for all $x \in N, x \neq 0$, a positive integer $n$ exists (in general, the number $n$ depends on the element $x$ ) such that $f^{n}(x)=0$. A perturbation of a DGA-module $N$ is a morphism of graded modules $\delta: N \rightarrow N$ of degree -1 , such that $\left(d_{N}+\delta\right)^{2}=0$ and $\xi_{N} \delta=0$. A perturbation datum of the contraction $r:\{N, M, f, g, \phi\}$ is a perturbation $\delta$ of the DGA-module $N$ verifying the condition that the composition $\phi \delta$ is pointwise nilpotent.

We now introduce the main tool in Homological Perturbation Theory: the Basic Perturbation Lemma ([6], [8], [7], [1]).

\section{THEOREM 2.1 (BPL)}

Let $r:\{N, M, f, g, \phi\}$ be a contraction and $\delta: N \rightarrow N$ a perturbation datum of $r$. Then, a new contraction

$$
r_{\delta}:\left\{\left(N, d_{N}+\delta, \epsilon_{N}, \eta_{N}\right),\left(M, d_{M}+d_{\delta}, \epsilon_{M}, \eta_{M}\right), f_{\delta}, g_{\delta}, \phi_{\delta}\right\}
$$

is defined by the formulas: $d_{\delta}=f \delta \Sigma_{r}^{\delta} g ; f_{\delta}=f\left(1-\delta \Sigma_{r}^{\delta} \phi\right) ; g_{\delta}=\Sigma_{r}^{\delta} g ; \phi_{\delta}=\Sigma_{r}^{\delta} \phi$; where

$$
\Sigma_{r}^{\delta}=\sum_{i \geq 0}(-1)^{i}(\phi \delta)^{i}=1-\phi \delta+\phi \delta \phi \delta-\cdots+(-1)^{i}(\phi \delta)^{i}+\cdots
$$

Let us note that $\Sigma_{r}^{\delta}(x)$ is a finite sum for each $x \in N$, because of the pointwise nilpotency of the composition $\phi \delta$. Moreover, it is obvious that the morphism $d_{\delta}$ is a perturbation of the DGA-module $\left(M, d_{M}, \epsilon_{M}, \eta_{M}\right)$.

The perturbation problem for more complex structures has been extensively studied. The most significant results for DGA-algebras (or DGA-coalgebras) can be found in [8], [9] and [18]. First, we review several notions.

Definition 2.2 [18] Let $A$ and $A^{\prime}$ be two DGA-algebras and $r:\left\{A, A^{\prime}, f, g, \phi\right\}$ a contraction. The projection $f$ is a quasi algebra projection if the following conditions hold:

$$
\begin{gathered}
f \mu_{A}(\phi \otimes \phi)=0, \quad f \mu_{A}(\phi \otimes g)=0, \\
f \mu_{A}(g \otimes \phi)=0,
\end{gathered}
$$

The homotopy operator $\phi$ is a a quasi algebra homotopy if the following conditions hold:

$$
\begin{gathered}
\phi \mu_{A}(\phi \otimes \phi)=0, \quad \phi \mu_{A}(\phi \otimes g)=0, \\
\phi \mu_{A}(g \otimes \phi)=0,
\end{gathered}
$$

Definition 2.3 [8] Let $A$ and $A^{\prime}$ be two DGA-algebras and $r:\left\{A, A^{\prime}, f, g, \phi\right\}$ a contraction. The homotopy operator $\phi$ is said to be an algebra homotopy if

$$
\phi \mu_{A}=\mu_{A}\left(1_{A} \otimes \phi+\phi \otimes g f\right) .
$$


Definition 2.4 [18] Let $A$ and $A^{\prime}$ be two DGA-algebras and $r:\left\{A, A^{\prime}, f, g, \phi\right\}$ a contraction. We say that $r$ is

- a semi-full algebra contraction if $f$ is a quasi algebra projection, $g$ is a morphism of DGA-algebras and $\phi$ is a quasi algebra homotopy.

- an almost-full algebra contraction if $f$ and $g$ are morphisms of DGA-algebras and $\phi$ is a quasi algebra homotopy.

- a full algebra contraction if $f$ and $g$ are morphisms of DGA-algebras and $\phi$ is an algebra homotopy.

Obviously, full and almost-full algebra contractions are, in particular, semi-full algebra contractions. It is not difficult to prove that both sets of semi-full and almostfull algebra contractions are closed by composition and tensor product of contractions.

Definition 2.5 [7] Let $A$ and $A^{\prime}$ be two DGA-algebras and $r:\left\{A, A^{\prime}, f, g, \phi\right\}$ a contraction. An algebra perturbation datum $\delta$ of $r$ is a perturbation datum of this contraction which is also a derivation.

The following result tells us that the set of semi-full algebra contractions is closed by homological perturbation. This theorem plays a key role in the proof of the main theorem of this paper.

\section{THEOREM 2.6 (SF-APL) ([18])}

Taking as data a semi-full algebra contraction $r$ and an algebra perturbation datum $\delta$ of $r$, the perturbed contraction $r_{\delta}$ is an algebra contraction of the same type, where the product on $A_{\delta}^{\prime}$ is the original product $\mu_{A^{\prime}}$.

\section{Some semi-full algebra contractions}

In this section, we give several algebra contractions which will be essential in the next section. However, we do not explicitly describe the morphisms of these contractions because this is not relevant in the sequel.

The following functions will be extremely useful:

Definition 3.7 [3] Let $A$ be a DGA-algebra. We define the following morphisms of graded modules:

- The suspension, $\sigma: A \rightarrow \bar{B}(A)$ defined by $\sigma(a)=[a]$, where $a \in A$.

- The p-transpotence, where $p$ is a prime number, $\varphi_{p}: A \rightarrow \bar{B}(A)$, defined by $\varphi_{p}(a)=\left[a \mid a^{p-1}\right]$ where $a \in A$. 
Notice that Cartan defined the homology operations of suspension and $p$-tranpotence while we define here analogous functions from a DGA-algebra $A$ to its reduced bar construction.

We now analyze an important contraction "connecting" the reduced bar contruction of a tensor product of two DGA-algebras and the tensor product of reduced bar constructions of these DGA-algebras.

Proposition 3.8 [5] Let $A$ and $A^{\prime}$ be two commutative DGA-algebras. There is a contraction, that we denote by $R_{b \otimes}$, from $\bar{B}\left(A \otimes A^{\prime}\right)$ to $\bar{B}(A) \otimes \bar{B}\left(A^{\prime}\right)$

Eilenberg and Mac Lane also proved in [5] that the projection and the inclusion of the contraction $R_{b \otimes}$ are morphisms of DGA-algebras. The fact that the homotopy operator of this contraction is a quasi algebra homotopy and, therefore, that the last contraction is an almost-full algebra contraction is proved in [18].

Let $\otimes_{i \in I} A_{i}$ be a tensor product of commutative DGA-algebras. Using $R_{b \otimes}$ and classical contraction constructions, we can determine a contraction from $\bar{B}\left(\otimes_{i \in I} A_{i}\right)$ to $\otimes_{i \in I} \bar{B}\left(A_{i}\right)$. We also denote this contraction by $R_{b \otimes}$.

Other interesting contractions in the sequel are:

- ([5]) An isomorphism of Hopf algebras, that we denote by $R_{b e}$, from $\bar{B}(E(u, 2 n-$ 1)) to $\Gamma(\sigma(u), 2 n)$. Obviously, this datum can be seen as a full algebra contraction.

- ([5], [18]) A contraction, that we denote by $R_{b q}$, from $\bar{B}\left(Q_{p}(v, 2 n)\right)$ to $E(\sigma(v), 2 n+1) \otimes \Gamma\left(\varphi_{p}(v), 2 n p+2\right)$ which is an almost-full algebra contraction. $Q_{p}(v, 2 n)$ denotes the truncated polynomial algebra $P(v, 2 n) /\left(v^{p}\right)$.

We have denoted the generators on the small algebras of the two last contractions by making use of the suspension and $p$-transpotence functions. For instance, the generator $v$ of the divided power algebra in the contraction $R_{b e}$ has been denoted by $\sigma(u)$. This means that the image of $v$ by the inclusion of this contraction is $\sigma(u)$.

\section{A generalization of a result of Moore}

We are mainly concerned with the fact of obtaining, working over $\mathbb{Z}_{(p)}$, homological models of a special type of commutative DGA-algebras.

First, an important notion is introduced: twisted tensor product of commutative DGA-algebras.

Definition 4.9 Let $\left\{A_{i}\right\}_{i \in I}$ and $\rho$ be a family of commutative DGA-algebras and a derivation of degree -1 of the DGA-algebra $\otimes_{i \in I} A_{i}$. A twisted tensor product (or TTP) $\tilde{\otimes}_{i \in I}^{\rho} A_{i}$ is a commutative DGA-algebra satisfying the following conditions: 
- i) as graded algebra, $\tilde{\otimes}_{i \in I}^{\rho} A_{i}$ coincides with the tensor product $\otimes_{i \in I} A_{i}$,

- ii) and its differential is the sum of the differential of the ordinary tensor product and the derivation $\rho$.

Then a TTP of DGA-algebras is a perturbed DGA-algebra where the "perturbation" affects its differential (and not its product). Notice that this notion shows a little structural difference with respect to that of multiplicative principal construction of Cartan (see [3], [16]). Notice that the third and fourth Cartan's elementary complexes are TTPs of DGA-algebras and, at the same time, they are twisted tensor products (in Brown's sense [2]).

We are interested here in a particular class of free resolutions of $\Lambda$ over a commutative DGA-algebra $A$, those that are TTPs of the form $X=A \tilde{\otimes}^{\rho} \bar{X}$, where $\bar{X}$ is a free commutative DGA-algebra. $\bar{X}$ is the reduced complex of the resolution. For instance, if $A$ is a commutative DGA-algebra, the bar resolution $B(A)$ becomes a twisted tensor product of DGA-algebras. More precisely, $B(A)$ is the commutative DGA-algebra $A \tilde{\otimes}^{\rho} \bar{B}(A)$, where

$$
\rho\left(a \otimes\left[a_{1}\left|a_{2}\right| \cdots \mid a_{n}\right]\right)=\mu_{A}\left(a, a_{1}\right) \otimes\left[a_{2}|\cdots| a_{n}\right] .
$$

In this case, the contraction $R_{B(A)}$ (see (1)) is an almost-full algebra contraction.

Definition 4.10 A twisted tensor product $T T P=\tilde{\otimes}_{i \in I}^{\rho} A_{i}$ is called decomposable if there is a non-trivial partition $I=I_{1} \cup I_{2}$ of $I$, such that TTP decomposes into a tensor product of twisted tensor products $T T P_{1}=\tilde{\otimes}_{i \in I_{1}}^{\rho_{1}} A_{i}$ and $T T P_{2}=\tilde{\otimes}_{i \in I_{2}}^{\rho_{2}} A_{i}$ with $\rho_{m}=\left.\rho\right|_{T T P_{m}}$ for $m=1,2$. Otherwise, TTP is called indecomposable of length $\ell$ (or $\ell$-indecomposable), where $\ell$ is the cardinal of the set of indices $I$.

Obviously, Cartan's elementary complexes are 1-indecomposable and 2-indecomposable TTPs.

In this section, we determine an algebra contraction from the reduced bar construction of a TTP A of Cartan's elementary complexes to a free commutative DGA-algebra $H$. This information can be used to derive small free resolutions of $\Lambda$ over $A$ as it is shown in the following theorem, which is a graded-commutative version of Theorem 8.1 .3 of $[11]$ :

THEOREM 4.11 Let $A$ be a connected commutative DGA-algebra. Given a semi-full algebra contraction from the reduced bar construction $\bar{B}(A)$ to a free commutative DGAalgebra $H$, in which the homotopy operator increases the simplicial degree by one, there is a free resolution $K$ which split off of the bar construction. Moreover, this resolution $K$ is a twisted tensor product of the DGA-algebras $A$ and $H$ and the splitting is a semi-full algebra contraction. 
Proof.

First of all, given a semi-full algebra contraction $r:\{\bar{B}(A), H, f, g, \phi\}$, it is possible to construct the tensor product contraction

$$
1_{A} \otimes r:\left\{A \otimes \bar{B}(A), \quad A \otimes H, 1_{A} \otimes f, 1_{A} \otimes g, 1_{A} \otimes \phi\right\}
$$

It is clear that the previous contraction is a semi-full algebra contraction, taking into account the natural products on $A \otimes \bar{B}(A)$ and $A \otimes H$.

Now, the question boils down to applying the SF-APL Theorem to this contraction, taking as algebra perturbation datum the morphism $\rho$ (see (2)). Obviously, $\rho$ is a derivation-perturbation of $A \otimes \bar{B}(A)$. It remains to prove the pointwise nilpotency of the composition $\left(1_{A} \otimes \phi\right) \rho$. The DGA-algebra $A \otimes \bar{B}(A)$ inherits a filtration given by the tensor degree of the reduced bar construction $\bar{B}(A)$. It is easy to see that $\rho$ lowers filtration, at least, by one (notice that $A$ is connected). On the other hand, since $1_{A} \otimes \phi$ increases simplicial degree by one, this homotopy operator is filtration preserving. Then, $\left(1_{A} \otimes \phi\right) \rho$ lowers the filtration, at least, by one, and this means that this composition is pointwise nilpotent. This completes the proof.

From now on, the ground ring will be $\mathbb{Z}_{(p)}$. Notice that this restriction will be essential in the achievement of good homological results in the sequel.

Definition 4.12 [9] Let $M$ be a DGA-module over $\mathbb{Z}_{(p)}$. We say that a morphism of DGA-modules $h: M \rightarrow M$ is $p$-minimal if $h(M) \subseteq p \cdot M$. We say a DGA-module $M$ is $p$-minimal if it is free and of finite type as graded module over $\mathbb{Z}_{(p)}$, and its differential $d_{M}$ is $p$-minimal. It is known that a contraction between two $p$-minimal DGA-modules is an isomorphism of DGA-modules.

Definition 4.13 We say that a commutative DGA-algebra $H$ is a $p$-minimal homological model of a commutative DGA-algebra $A$ if $H$ is $p$-minimal and there is an (explicit) semi-full algebra contraction from the reduced bar construction $\bar{B}(A)$ to $H$.

In [18] it is established that $p$-minimal homological models of Cartan's elementary complexes are, in their turn, tensor products of elementary complexes. In this section, not only do we want to determine the algebras making up $p$-minimal homological models of TTPs of Cartan's elementary complexes, but we also want to control the behavior of the differentials on these models. In order to prove this result, we need several theorems of [18]. First of all, an explicit algebra contraction for $\bar{B}(\Gamma(u, 2 n))$ is constructed by perturbation. More precisely, in this case we apply the SF-APL Theorem to a tensor product of contractions $R_{b q}$. Here we will only deal with odd primes $p$. For $p=2$, analogous results are obtained. From now on, we denote the 
generators of the small DGA-algebras of the contractions in relation to the functions of suspension $\sigma, p$-transpotence $\varphi_{p}$ and $k$-th divided power $\gamma_{k}$.

THEOREM 4.14 ([18]) There is a semi-full algebra contraction $R_{b \Gamma}$ from $\bar{B}(\Gamma(u, 2 n))$ to a tensor product of the exterior algebra $E(\sigma(u), 2 n+1)$ and 2-indecomposable twisted tensor products of the form

$$
E\left(\sigma \gamma_{p^{i}}(u), 2 n p^{i}+1\right) \tilde{\otimes}^{\rho_{p}} \Gamma\left(\varphi_{p} \gamma_{p^{i-1}}(u), 2 n p^{i}+2\right) .
$$

We also get in [18] the following semi-full algebra contractions:

(a) from $\bar{B}\left(E(u, 2 n-1) \tilde{\otimes}^{\rho_{ \pm p} r} \Gamma(v, 2 n)\right)$ to a tensor product

$$
\begin{gathered}
{\left[\Gamma(\sigma(u), 2 n) \tilde{\otimes}^{\rho_{\mp p^{r}}} E(\sigma(v), 2 n+1)\right] \otimes} \\
\left(\otimes_{i \geq 1}\left[E\left(\sigma \gamma_{p^{i}}(v), 2 n p^{i}+1\right) \tilde{\otimes}^{\rho_{p}} \Gamma\left(\varphi_{p} \gamma_{p^{i-1}}(v), 2 n p^{i}+2\right)\right]\right) .
\end{gathered}
$$

(The notation $\sigma \widetilde{\gamma_{p^{i}}}(v)$ means that a change of basis has been done in which the generator of the algebra $\left.E\left(\sigma \gamma_{p^{i}}(v), 2 n p^{i}+1\right)\right)$ has been modified.)

(b) from $\bar{B}\left(\Gamma(u, 2 n) \tilde{\otimes}^{\rho_{ \pm p^{r}}} E(v, 2 n+1)\right)$ to a tensor product of the form

$$
\begin{gathered}
{\left[E(\sigma(u), 2 n+1) \tilde{\otimes}^{\rho_{\mp p^{r}}} \Gamma(\sigma(v), 2 n+2)\right] \otimes} \\
\left(\otimes_{i \geq 1}\left[E\left(\sigma \gamma_{p^{i}}(u), 2 n p^{i}+1\right) \tilde{\otimes}^{\rho_{p}} \Gamma\left(\varphi_{p} \gamma_{p^{i-1}}(u), 2 n p^{i}+2\right)\right]\right) .
\end{gathered}
$$

In the case (a), a basis change is necessary to get a tensor product of 2indecomposable twisted tensor product as $p$-minimal homological model. This idea was already used by Moore in [15].

In order to complete our homological study of 2-indecomposable $p$-minimal TTPs of exterior and divided power algebras, we must analyze the case of the DGA-algebra $A=\Gamma(u, 2 n) \tilde{\otimes}^{\rho^{\prime}} E(v, 2 n t+1)$ (where $\left.\rho^{\prime}(v)= \pm p^{s} \gamma_{t}(u), s, t \in \mathbf{N}\right)$. Taking into account the techniques of [18], it is easy to deduce a semi-full algebra contraction from $\bar{B}(A)$ to a tensor product of indecomposable $p$-minimal TTPs of length 1 and 2. For instance, if $t=p^{r}$, we have the following $p$-minimal homological model

$$
\begin{gathered}
E(\sigma(u), 2 n+1) \otimes \Gamma\left(\widetilde{\sigma(v)}, 2 n p^{r}+2\right) \otimes \\
\left(\otimes_{i \geq 1}\left[E\left(\sigma \gamma_{p^{i}}(u), 2 n p^{i}+1\right) \tilde{\otimes}^{\rho_{p}} \Gamma\left(\varphi_{p} \gamma_{p^{i-1}}(u), 2 n p^{i}+2\right)\right]\right) .
\end{gathered}
$$

To sum up, these particular indescomposable TTPs of length 1 and 2 have as $p$-minimal homological models, TTPs of the same type. Theorem 4.11 allows us to translate these results into the lenguage of resolutions and, in this way, we recover the Moore's work in [15].

Finally, the problem of generalizing these results to indecomposable TTPs of length higher than 2 is solved in the following theorem, which tells us that there is a $p$-local homological non-degeneration result of $\ell$-indecomposability $(\ell \geq 2)$. 
THEOREM 4.15 There is a semi-full algebra contraction from the reduced bar construction of a $\ell$-indecomposable (with $\ell \geq 2$ ) p-minimal TTP A of exterior and divided power algebras to a tensor product $H$ of $k$-indecomposable (with $k \leq \ell$ ) p-minimal TTPs of exterior and divided power algebras (equipped with its natural product). That is, $H$ is a p-minimal homological model of $A$.

Moreover, at the graded algebra level, we have that

- each $E(u, 2 n-1)$ factor in $A$ contributes with a $\Gamma(\sigma(u), 2 n)$ factor in $H$.

- each $\Gamma(u, 2 n)$ factor in $A$ contributes with $E\left(\sigma \gamma_{p^{i}}(u), 2 n p^{i}+1\right)$ (with $\left.i \geq 0\right)$ and $\Gamma\left(\varphi_{p} \gamma_{p^{i-1}}(u), 2 n p^{i}+2\right)$ (with $\left.i \geq 1\right)$ factors in $H$.

Proof.

If $A$ is a DGA-algebra and $d: A \rightarrow A$ is a differential and a derivation, the notation $(A, d)$ means a DGA-algebra such that its graded algebra structure is that of $A$ and its differential is $d$. If $A$ is a connected DGA-algebra, Ker $\epsilon_{A}$ in degree $n$ is $A_{n}$ if $n>0$ and 0 if $n=0$. In this case, we denote $\operatorname{Ker} \epsilon_{A}$ by $\bar{A}$

Using the previous contractions and applying perturbation, it is an easy task to establish the construction process of an explicit algebra contraction for the reduced bar construction of any twisted tensor product $A=\tilde{\otimes}_{i \in\{1, \ldots, \ell\}}^{\rho} A_{i}$, where $A_{i}=E\left(u_{i}, n_{i}\right)$ (with $n_{i}$ odd) or $A_{i}=\Gamma\left(u_{i}, n_{i}\right)$ (with $n_{i}$ even) and $n_{1} \leq n_{2} \leq \cdots \leq n_{\ell}$. We use the contractions $R_{b \otimes}, R_{b e}, R_{b \Gamma}$ to construct a semi-full algebra contraction $R$ from $\bar{B}\left(\otimes_{i=1}^{\ell} A_{i}\right)$ (excluding $\rho$ ) to a tensor product $\left(H, d_{H}\right)=\otimes_{i=1}^{\ell} H_{i}$, where $H_{i}=\Gamma(\sigma(u), 2 n)$ if $A_{i}=E(u, 2 n-1)$ and

$$
H_{i}=E(\sigma(u), 2 n+1) \otimes \otimes_{i \geq 1}\left[E\left(\sigma \gamma_{p^{i}}(u), 2 n p^{i}+1\right) \tilde{\otimes}^{\rho_{p}} \Gamma\left(\varphi_{p} \gamma_{p^{i-1}}(u), 2 n p^{i}+2\right)\right]
$$

if $A_{i}=\Gamma(u, 2 n)$. From now on, we omit the degree of the generator in the description of the algebras. The $p$-minimal derivation $\rho$ produces an algebra perturbation datum $\delta$ for $R$. More concretely, $\rho$ induces a perturbation datum in the tensor differential of $\bar{B}\left(\otimes_{i \in I} A_{i}\right)$. It is clear that $\delta$ is a $p$-minimal derivation. Now, let us denote by $f, g$ and $\phi$ the projection, inclusion and homotopy operator of $R$. We deduce the pointwise nilpotency of the composite $\phi \delta$ from the following facts:

- $\phi$ increases simplicial degree in $\bar{B}\left(\otimes_{i \in I} A_{i}\right)$ by 1 .

- $\delta$ does not change simplicial degree. In fact, this morphism decreases tensor degree by 1 .

It is time to apply SF-APL, obtaining in this way a perturbed semi-full algebra contraction $R_{\delta}$ from $\bar{B}(A)$ to $\left(H, d_{H}+d_{\delta}\right)$. Keeping in mind that $\delta$ is $p$-minimal, it is clear that the small DGA-algebra $\left(H, d_{H}+d_{\delta}\right)$ of $R_{\delta}$ is a $p$-minimal homological model of $A$. The fact that the inclusion of $R_{\delta}$ is a morphism of DGA-algebras induces an 
enormous calculational improvement in the determination of the perturbed differential on $H$. The presence of a DGA-algebra structure in the $p$-minimal homological model severely limits how the differential $d_{\delta}=f \delta \Sigma_{r}^{\delta} g$ can act. In fact, we only need to compute this morphism on the generators of this DGA-algebra.

Therefore, the graded algebra structure of the $p$-minimal homological model has been determined. Now, we analyze its differential structure in more detail. We prove by a straightforward induction on number of DGA-algebras that a $p$-minimal homological model of $A$ can be a tensor product of $k$-indecomposable twisted tensor products, with $k \leq \ell$. Now we apply the perturbation machinery $\ell-1$ times to $R$ by using the derivations $\left.\delta\right|_{\otimes_{i=1}^{k} A_{i}}, 2 \leq k \leq \ell$. We control the behavior of the perturbed differential in the $p$-minimal homological model using change-of-basis arguments as in the 2-indecomposable case (a).

First, we state a result that is the key to establish the homological preservation (or, more precisely, non-degeneration) of the $\ell$-indecomposability of the TTP $A$. The proof is left to the reader.

Let $\left\{C_{i}\right\}_{i \in I}$ be a family of Cartan's elementary complexes of the form $E(u) \tilde{\otimes}^{\rho_{ \pm p}} \Gamma(v)$ with differential operator defined by $\rho_{ \pm p}(v)= \pm p u$ and $\rho_{ \pm p}(u)=0$. If we consider the DGA-algebra

$$
C=\otimes_{i \in I} C_{i},
$$

it is not difficult to prove the following property:

$$
p \operatorname{Ker} d_{C}=\operatorname{Im} d_{C} \text {. }
$$

Moreover, if $B$ is a $p$-minimal TTP of exterior and divided power algebras and we denote the differential of $B \otimes \bar{C}$ by $d_{B C}$, we have:

$$
p \operatorname{Ker} d_{B C}=\operatorname{Im} d_{B C} .
$$

After these preliminaries, it is time to apply induction on number of DGA-algebras. For $\ell=2$, all has been proved.

The DGA-algebra $A=\tilde{\otimes}_{i \in\{1, \ldots, \ell\}}^{\rho} A_{i}$ can be expressed in the form

$$
A=\tilde{\otimes}_{i \in\{1, \ldots, \ell-1\}}^{\rho_{\ell-1}^{\prime}} A_{i} \tilde{\otimes}^{\rho_{\ell}} A_{\ell}
$$

where $\rho_{\ell-1}^{\prime}=\left.\rho\right|_{\otimes_{i=1}^{\ell-1} A_{i}}$ and $\rho_{\ell}=\left.\rho\right|_{A_{\ell}}$. It is clear that $\tilde{\otimes}_{i \in\{1, \ldots, \ell-1\}}^{\rho_{\ell-1}^{\prime}} A_{i}$ is a $p$-minimal TTP of $\ell-1$ algebras. Our induction hypothesis is that there is a semi-full algebra contraction, denoted by $R_{\ell-1}$, from the reduced bar construction of this last algebra to a tensor product $\left(H_{\ell-1}^{\prime}, d^{\prime}\right)$ of two DGA-algebras $\left(H_{\ell-1,1}^{\prime}, d_{1}^{\prime}\right)$ and $\left(H_{\ell-1,2}^{\prime}, d_{2}^{\prime}\right)$. The former $\left(H_{\ell-1,1}^{\prime}, d_{1}^{\prime}\right)$ is a tensor product of $\ell_{r}$-indecomposable $p$-minimal TTPs, with $r \in\{1, \ldots, s\}$ and $\sum_{r=1}^{s} \ell_{r}=\ell-1$. The exterior and divided power algebras that make up this tensor product are all the algebras $E\left(\widehat{\sigma\left(u_{i}\right)}\right)$ and $\Gamma\left(\widehat{\sigma\left(u_{i}\right)}\right)$ from the $p$-minimal 
homological model of $\otimes_{i=1}^{\ell-1} A_{i}$. The notation $D(\hat{u})$, where $D$ is the symbol $E$ or $\Gamma$, means that a basis change may have been done in the process, such that the generator of the algebra $D(u)$ could have been modified.

On the other hand, $\left(H_{\ell-1,2}^{\prime}, d_{2}^{\prime}\right)$ is a DGA-algebra as the one described in (3), composed by the pairs

$$
E\left(\sigma \gamma_{p^{j}}\left(u_{i}\right)\right) \tilde{\otimes}^{\rho_{p}} \Gamma\left(\varphi_{p} \gamma_{p^{j-1}}\left(u_{i}\right)\right)
$$

(where $u_{i}$ is of even degree) of the $p$-minimal homological model of $\otimes_{i=1}^{\ell-1} A_{i}$.

By using the contractions $R_{\ell-1}, R_{b \otimes}, R_{b e}$ and $R_{b \Gamma}$, we construct a semi-full algebra contraction, that we denote by $R_{\ell}$, from $\bar{B}\left(\tilde{\otimes}_{i \in\{1, \ldots, \ell-1\}}^{\rho_{\ell-1}^{\prime}} A_{i} \otimes A_{\ell}\right)$ to $\left(H_{\ell-1}^{\prime}, d^{\prime}\right) \otimes H_{\ell}$. Taking as perturbation datum the derivation $\delta_{\ell}$ produced by $\rho_{\ell}$ in the tensor diferencial of $\bar{B}\left(\tilde{\otimes}_{i=1, \ldots, \ell-1}^{\rho_{\ell-1}^{\prime}} A_{i} \otimes A_{\ell}\right)$, we perturb the contraction $R_{\ell}$ and we get a new semi-full algebra contraction $\left(R_{\ell}\right)_{\delta_{\ell}}$ from $\bar{B}(A)$ to the TTP $\left(H_{\ell-1}^{\prime}, d^{\prime}\right) \tilde{\otimes}^{d_{\delta_{\ell}}} H_{\ell}$. We also denote the latter by $(H, d)$. The differential $d_{\delta_{\ell}}$ is determined by its action on the generators of the DGA-algebra $H_{\ell}$. If $A_{\ell}=E\left(u_{\ell}\right)$, then $H_{\ell}=\Gamma\left(\sigma\left(u_{\ell}\right)\right)$. In this case, we only need to compute the differential on the generator $\sigma\left(u_{\ell}\right)$. Suppose that

$$
d_{\delta_{\ell}}\left(\sigma\left(u_{\ell}\right)\right)=p^{a} v+p^{b} w,
$$

where $a, b \in \mathbf{N}, v \in H_{\ell-1,1}^{\prime}$ and $w \in H_{\ell-1,1}^{\prime} \otimes \overline{H_{\ell-1,2}^{\prime}}$. Since $d^{\prime} d_{\delta_{\ell}}\left(\sigma\left(u_{\ell}\right)\right)=0$, then

$$
p^{a} d_{1}^{\prime}(v)+p^{b} d^{\prime}(w)=0 .
$$

From the facts $d_{1}^{\prime}(v) \in H_{\ell-1,1}^{\prime}$ and $d^{\prime}(w) \in H_{\ell-1,1}^{\prime} \otimes \overline{H_{\ell-1,2}^{\prime}}$, we deduce that $d_{1}^{\prime}(v)=0$ and $d^{\prime}(w)=0$. By the property (4), there is an element $\underline{w} \in H_{\ell-1,1}^{\prime} \otimes \overline{H_{\ell-1,2}^{\prime}}$, such that $d^{\prime}(\underline{w})=p w$.

If we change the generator $\sigma\left(u_{\ell}\right)$ into the generator $\widetilde{\sigma\left(u_{\ell}\right)}=\sigma\left(u_{\ell}\right)-p^{b-1} w$, we have $d\left(\widetilde{\sigma\left(u_{\ell}\right)}\right)=d_{\delta_{\ell}}\left(\widetilde{\sigma\left(u_{\ell}\right)}\right)=p^{a} v$, and, consequently, we see that a $p$-minimal homological model of $A$ is a tensor product of $k$-indecomposable $p$-minimal TTP (with $k \leq \ell$ ) of exterior and divided power algebras.

If $A_{\ell}=\Gamma\left(u_{\ell}\right)$ then

$$
H_{\ell}=E\left(\sigma\left(u_{\ell}\right)\right) \otimes\left(\otimes_{i=1}^{\infty}\left[E\left(\sigma \gamma_{p^{i}}\left(u_{\ell}\right)\right) \tilde{\otimes}^{\rho_{p}} \Gamma\left(\varphi_{p} \gamma_{p^{i-1}}\left(u_{\ell}\right)\right)\right]\right),
$$

and so, we must determine the differential $d_{\delta_{\ell}}$ on the generators $\sigma\left(u_{\ell}\right), \sigma \gamma_{p^{i}}\left(u_{\ell}\right)$ and $\varphi_{p} \gamma_{p^{i-1}}\left(u_{\ell}\right)$, for all $i=1,2, \ldots$. The process described in the previous case for the generator $\sigma\left(u_{\ell}\right)$ can also be done here and we obtain that the differential $d_{\delta_{\ell}}$ on this generator (up to basis change) lies in ${H^{\prime}}_{\ell-1}^{1}$.

Now, we control the behavior of $d_{\delta_{\ell}}$ on the couples of generators $\sigma \gamma_{p^{i}}\left(u_{\ell}\right)$ and $\varphi_{p} \gamma_{p^{i-1}}\left(u_{\ell}\right)$, for $i \geq 1$.

Suppose that 


$$
\begin{aligned}
d_{\delta_{\ell}}\left(\sigma \gamma_{p^{i}}\left(u_{\ell}\right)\right) & =p^{a} v_{i}, \\
d_{\delta_{\ell}}\left(\varphi_{p} \gamma_{p^{i-1}}\left(u_{\ell}\right)\right) & =p^{b} w_{i},
\end{aligned}
$$

with $a, b \in \mathbf{N}$ and $v_{i}, w_{i} \in H, \forall i \geq 1$.

On the other hand, we have that

$$
\begin{aligned}
& d\left(\sigma \gamma_{p^{i}}\left(u_{\ell}\right)\right)=d_{\delta_{\ell}}\left(\sigma \gamma_{p^{i}}\left(u_{\ell}\right)\right) \\
& \rho_{p}\left(\varphi_{p} \gamma_{p^{i-1}}\left(u_{\ell}\right)\right)=p \sigma \gamma_{p^{i}}\left(u_{\ell}\right)
\end{aligned}
$$

and

$$
d\left(\varphi_{p} \gamma_{p^{i-1}}\left(u_{\ell}\right)\right)=\left(\rho_{p}+d_{\delta_{\ell}}\right)\left(\varphi_{p} \gamma_{p^{i-1}}\left(u_{\ell}\right)\right)
$$

If we change the generator $\sigma \gamma_{p^{i}}\left(u_{\ell}\right)$ into the element $\sigma \widetilde{\gamma_{p^{i}}}\left(u_{\ell}\right)=\sigma \gamma_{p^{i}}\left(u_{\ell}\right)+p^{b-1} z_{i}$, we have that $d\left(\sigma \gamma_{p^{i}}\left(u_{\ell}\right)\right)=0$ and $d\left(\varphi_{p} \gamma_{p^{i-1}}\left(u_{\ell}\right)\right)=p \sigma \gamma_{p^{i}}\left(u_{\ell}\right)$. That is, after all these basis changes, the $p$-minimal homological model of $A$ is a tensor product of $k$-indecomposable $p$-minimal TTPs (with $k \leq \ell$ ) of exterior and divided power algebras.

This completes the proof of the theorem.

In the light of Theorem 4.11, a version of this result using resolutions instead of reduced complexes can be stated.

THEOREM 4.16 Let $\mathbb{Z}_{(p)}$ be the ground ring. Let $A$ be a $\ell$-indecomposable (with $\ell \geq 2$ ) p-minimal TTP of Cartan's elementary complexes. There is a free resolution $A \tilde{\otimes}^{\rho^{\prime}} H$ that split off of the bar construction. More precisely, the splitting is a semi-full algebra contraction and the reduced complex $H$ is determined by Theorem 4.15.

In this manner, we have established a generalization of Moore's work [15].

\section{References}

[1] D. W Barnes and L. A. Lambe. A fixed point approach to Homological Perturbation Theory. Proceeding of the A.M.S., vol. 112, n. 3 (1991) 881-892.

[2] E. H. Jr. Brown. Twisted tensor products I. Ann. Math. 69 (1959) 223-246.

[3] H. Cartan. Algèbres d'Eilenberg-Mac Lane. Séminaire H. Cartan 1954/55, (exposé 2 à 11), E. Normale Superiere, Paris, 1956. 
[4] S. Eilenberg and S. Mac Lane. On the groups $H(\pi, n)$, I. Annals of Math., 58 (1953) 55-139.

[5] S. Eilenberg and S. Mac Lane. On the groups $H(\pi, n)$, II. Annals of Math., 66 (1954) 49-139.

[6] V.K.A.M. Gugenheim. On the chain complex of a fibration. Illinois J. Math., 3 (1972) 398-414.

[7] V.K.A.M. Gugenheim and L. Lambe. Perturbation theory in Differential Homological Algebra, I. Illinois J. Math., vol. 33 (1989) 556-582.

[8] V.K.A.M. Gugenheim, L. Lambe and J.D. Stasheff. Perturbation theory in Differential Homological Algebra, II. Illinois J. Math., vol. 35, n. 3 (1991) 357-373.

[9] J. Huebschmann and T. Kadeishvili. Small models for chain algebras. Math. Z., vol. 207 (1991) 245-280.

[10] L. A. Lambe. Resolutions via homological perturbation.J. Symbolic Comp., 12 (1991) 71-87.

[11] L. A. Lambe. Homological perturbation theory, Hochschild homology and formal groups. Proc. Conference on Deformation Theory and Quantization with Applications to Physics, Amherst, MA, June 1990, Cont. Math., 134, A.M.S (1992) 183-218.

[12] L. A. Lambe. Resolutions which split off of the bar construction. Journal of Pure and Applied Algebra, 84 (1993) 311-329.

[13] S. Mac Lane. Homology. Classics in Matematics. Springer 1995.

[14] J. C. Moore. Cartan's constructions, the homology of $K(\pi, n)$ 's, and some later developments. Colloque "Analyse et Topologie" en l'Honneur de Henri Cartan (Orsay, 1974), Asterisque 32-33 (1976) 73-212.

[15] J. C. Moore. On the homology of $K(\pi, n)$. Proc. NAS. USA, 43 (1957) 409-411.

[16] A. Prouté. Algèbres différentielles fortement homotopiquement associatives $\left(A_{\infty}\right.$ algèbres). Ph. D. Thesis, Université Paris VII.

[17] P. Real. Sur les groupes d'homotopie. C. R. Acad. Sci. Paris, t. 319, série I (1994) $475-478$.

[18] P. Real. Homological Perturbation Theory and Associativity. Preprint n.: MA105-96I of the Dpto. Matematica Aplicada I, Sevilla (Spain) 1996.

[19] J. Stasheff. Deformation theory and the little constructions of Cartan and Moore. Collection: Algebraic Topology and algebraic K-theory (Princeton, NJ 1983), Ann. of Math Stud., 113 (1987) 322-331, Princeton Univ. Press, NJ. 
[20] J. Stasheff. Homotopy associativity of H-spaces, II. Transactions of A.M.S., vol. 108 (1963) 293-312. 